\title{
Impact of a pharmacy-led smoking cessation clinic in a dermatology centre
}

Hui Mei $\underline{\text { Cheng }}^{1}$, MBBS, Wen Chun Liu ${ }^{1}$, MBBS, MRCS, Germaine $\underline{\text { Chua }}^{1}$, BSc(Pharm)(Hons), Choon Fong Liew ${ }^{2}$, MBBS, MRCP, Winnie $\underline{\mathrm{Li}}^{1}$, BSc(Pharm), Winnie $\underline{\mathrm{ChoO}}^{1}$, BSc(Pharm)(Hons), Hazel H $\underline{\mathrm{O}}^{1}$, MD, MRCP

INTRODUCTION Cigarette smoking is a leading cause of morbidity and mortality, and has a deleterious effect on dermatological conditions, such as skin cancers, hidradenitis suppurativa and psoriasis. The study aimed to evaluate the efficacy of a pharmacist-led smoking cessation clinic in reducing cigarette smoking at a tertiary referral dermatology centre. We described the impact of this clinic to provide guidance on how such a model could be further improved and implemented more widely.

METHODS In this single-centre, retrospective study, 74 currently smoking patients who received counselling at a structured smoking cessation clinic between January 2010 and March 2013 were identified. Information on baseline demographic characteristics and detailed past medical history, including smoking history, was collected. Follow-up was conducted at two weeks and three months.

RESULTS At the first follow-up at two weeks, which was attended by 57 patients, 9 (15.8\%) had stopped smoking and $26(45.6 \%)$ showed reduction in the number of cigarette sticks smoked per day, with an average reduction of 4.1 cigarette sticks per day. However, a few patients also reported no change or increased number of cigarette sticks smoked per day following counselling.

CONCLUSION A structured pharmacist-led smoking cessation clinic is effective and can be made a part of the holistic management of dermatological conditions.

Keywords: dermatology, nicotine replacement therapy, smoking cessation, tobacco

\section{INTRODUCTION}

Cigarette smoking is a risk factor for various ailments, including cardiovascular disease, respiratory disease and malignancy. Lesser known are its effects on the integumentary system - an increasing body of evidence is pointing towards worse outcomes in patients with skin conditions among smokers, including wound healing and infection, skin cancers, hidradenitis suppurativa and psoriasis. ${ }^{(1-3)}$

A majority of smokers report a desire to quit smoking, with many attempting to quit. ${ }^{(4)}$ To this end, smoking cessation programmes have been developed to reduce the incidence and prevalence of cigarette smoking. This involves a trained individual skilled in behavioural counselling combined with pharmacological interventions to reduce the withdrawal symptoms experienced during the cessation period. ${ }^{(4)}$

Physical appearance interventions have a positive impact on both smoking-related cognition as well as cessation behaviour. Emphasising the effects of skin ageing and other detrimental effects of smoking on physical appearance were associated with better smoking cessation outcomes. ${ }^{(5)}$

The aim of the present study was to evaluate the efficacy of a structured smoking cessation clinic at a tertiary referral dermatology centre in reducing the number of cigarettes smoked per day.

\section{METHODS}

Since January 2010, smoking cessation clinics have been commenced at the National Skin Centre in Singapore, a tertiary referral dermatology centre. Four pharmacists were certified in a formal 'Quit Smoking' counselling course, where they learnt techniques of motivational interviewing to assist in counselling patients as well as the use of nicotine replacement therapy (NRT). Doctors recruited currently smoking patients from both general and subspecialty dermatology clinics who indicated an intention to quit smoking and were agreeable to counselling at the smoking cessation clinic. Written information reinforcing the health benefits of smoking cessation, with emphasis on skin health and physical appearance, was also dispensed to all participants. After entering the programme, follow-up was performed either faceto-face or via phone interview at two weeks and three months.

The study cohort comprised all participants who were newly recruited into the smoking cessation clinic from January 2010 to March 2013. Baseline demographic characteristics were collected. Past medical history, including dermatology diagnosis and treatment, and smoking history, including age of initiation of smoking, years of smoking and number of cigarettes smoked per day, number of quit attempts, time of last quit attempt, smokefree period for each attempt, previous methods used in the quit attempts (including use of NRT), and reasons for quitting were also collected. Further information collected during follow-up visits included adherence to NRT and number of cigarettes smoked.

Descriptive statistics were performed using Microsoft Excel 2013 (Microsoft Corp, Redmond, VA, USA) as per the per-protocol analysis. The study was approved by the ethics committee of the National Healthcare Group, Singapore. Patient consent was waived. 


\section{RESULTS}

Overall, 74 participants were identified, a majority of whom were Chinese $(52.7 \%)$ and male $(90.5 \%)$ patients. Mean patient age was 35.5 years. The average age of onset of smoking was 18.6 (range 9.0-55.0) years, with a smoking history of 12.8 (range 1.0-62.6) pack-years. Most (71\%) participants had attempted to quit in the past, with an average of two previous attempts to quit. The most often-cited reasons for quitting were health reasons $(81 \%)$, to improve relationships with family/friends (4\%), family health $(3 \%)$ and financial reasons (3\%). A majority of recruited participants had psoriasis (30\%), eczema (21\%) and discoid lupus erythematosus $(12 \%)$, with the remaining participants having a history of vitiligo, dermatophytic infection, acne vulgaris, chronic actinic dermatitis, immunobullous disorders, palmoplantar pustulosis, herpes infection, warts and alopecia (Fig. 1). Comorbidities included hyperlipidaemia $(n=12)$, hypertension $(n=8)$, diabetes mellitus $(n=4)$, and other cardiovascular $(n=3)$ or respiratory disorders $(n=4)$.

With regard to the choice of NRT, 11 (14.9\%) patients declined any NRT. The most commonly chosen NRT was the inhaler $(44.6 \%)$, followed by patch $(17.6 \%)$, gum $(13.5 \%)$ and lozenges $(9.5 \%)$.

At the first follow-up consultation at two weeks, 17 participants were lost to follow-up. Among the remaining 57 participants, 35 (61.4\%) either reduced the number of cigarette sticks smoked per day ( $\mathrm{n}=26 ; 45.6 \%$ ) or stopped smoking $(\mathrm{n}=9 ; 15.8 \%)$. Among the group who had reduced their smoking frequency, participants reported a reduction averaging 4.1 cigarette sticks per day. However, 18 (31.6\%) participants did not experience any reduction in smoking and a further $4(7.0 \%)$ participants reported an increase in their smoking pattern (Fig. 2).

At the final follow-up at three months, a further 30 participants were lost to follow-up. Among the remaining 27 patients, $9(33.3 \%)$ had stopped smoking, $6(22.2 \%)$ had a reduction of their cigarette consumption and $8(29.6 \%)$ had unchanged smoking frequency. However, 4 (14.8\%) participants reported an increase in smoking frequency (Fig. 3). Three of the four participants had showed an increase in cigarette consumption even at the first follow-up.

\section{DISCUSSION}

Our results showed that smoking cessation intervention at a dermatology clinic was effective. In a local study at a restructured government hospital, quit rates were $27.3 \%$ and $23.1 \%$ at three months of participation in the inpatient and outpatient smoking cessation programmes, respectively. ${ }^{(6)}$ Similarly, a recent Cochrane review demonstrated a quit rate of $18 \%-21 \%$, depending on whether behavioural support was administered with pharmacotherapy. ${ }^{(7)}$ Comparatively, in our study, $33.3 \%$ of participants ceased smoking and $22.2 \%$ had a reduction of cigarettes smoked at three months.

In our study, careful discussion of the various NRTs was performed with the patient, with a range of options being offered. This likely increased compliance rates to NRT and contributed to the effectiveness of smoking cessation counselling.

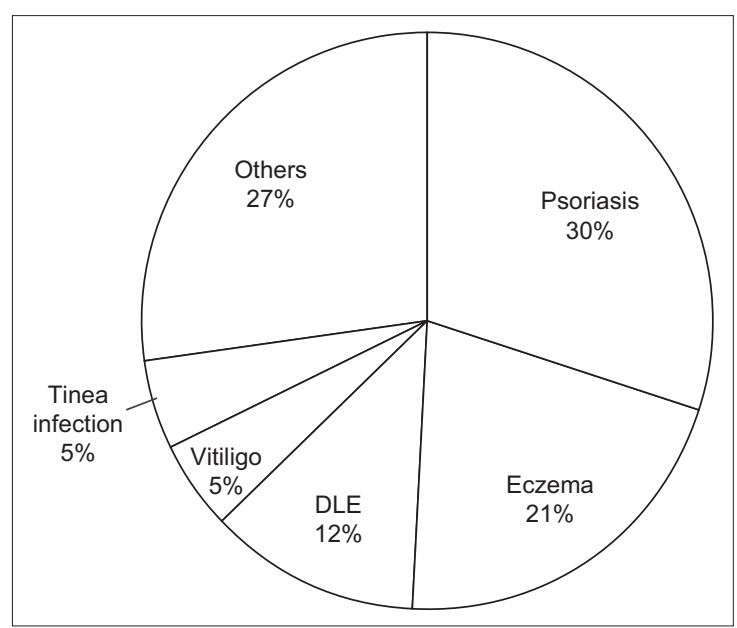

Fig. 1 Chart shows the dermatological conditions seen among study participants. DLE: discoid lupus erythematosus

One of the limitations of this study included its relatively short follow-up period of three months. However, habit formation tends to occur within this period, ${ }^{(8)}$ and this was consistent with other smoking cessation studies, where quit rates did not change significantly over time. ${ }^{(9,10)}$ There was also a high loss to follow-up, but this is a common problem in smoking cessation trials. ${ }^{(7)}$ In the present study, reasons cited for dropping out included participants having already quit and not seeing the need for further followup, or, conversely, that they had relapsed and were no longer motivated to quit. Some patients also cited time and financial constraints as limiting factors to compliance with follow-up. However, no participant indicated that loss to follow-up was due to any adverse events from the prescribed NRT or dissatisfaction with the programme.

Previous studies have found several factors to be associated with lower attrition rates, including older participant age, being followed up by telephone call, smoking relapse or failure to reduce smoking levels. ${ }^{(1)}$ To reduce dropout rates in future studies, strategies to engage younger participants should be followed and reassurances given to participants that smoking behaviour should not influence ongoing participation in the study. ${ }^{(11)}$ When participants are followed up by telephone calls, researchers should work flexible hours, so that those who are employed can be contacted during regular working hours. ${ }^{(11)}$ At our centre, we have reduced fees for follow-up appointments to reduce the financial load. In our study, participants commencing the programme were counselled that attending at least one follow-up was mandatory. Increasingly, more pharmacists are being trained to run the programme and doctors will receive prompts on the computer during consultation with participants, so that they can play a more constructive and hands-on role in motivational interviewing as well as encouraging compliance with treatment and follow-up.

Interestingly, four participants in our study experienced an increase in the number of cigarettes smoked after counselling. Although reasons for this increase were not investigated as part of the study, these four participants did not vary significantly from our study population, in general, in terms of baseline 


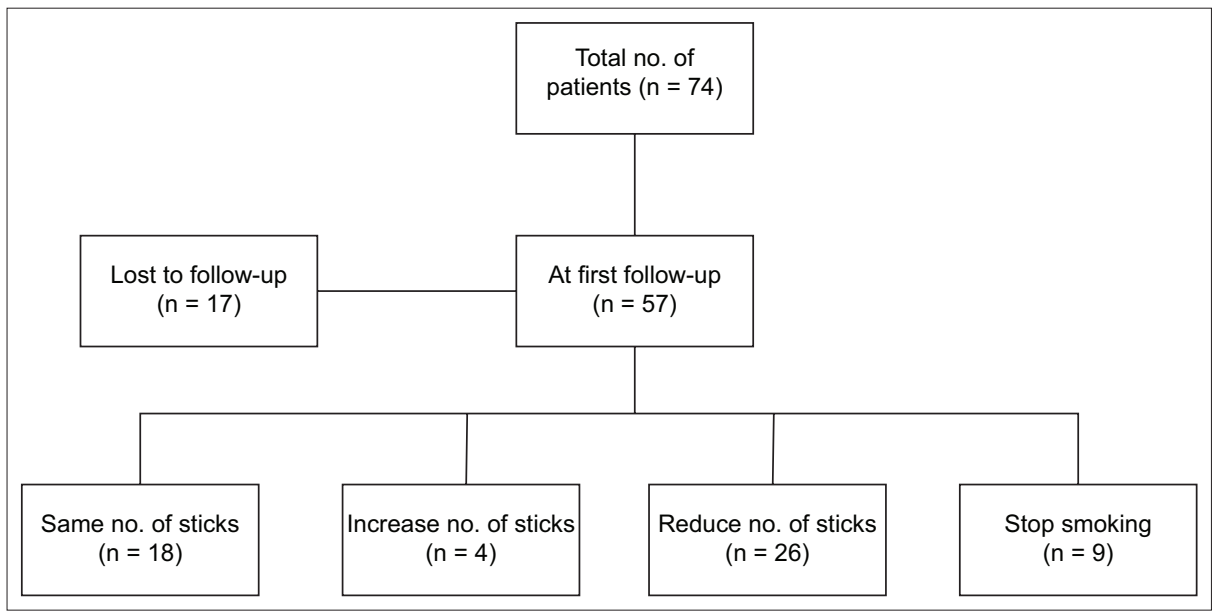

Fig. 2 Diagram shows the change in the number of cigarette sticks smoked per day at the first follow-up at two weeks.

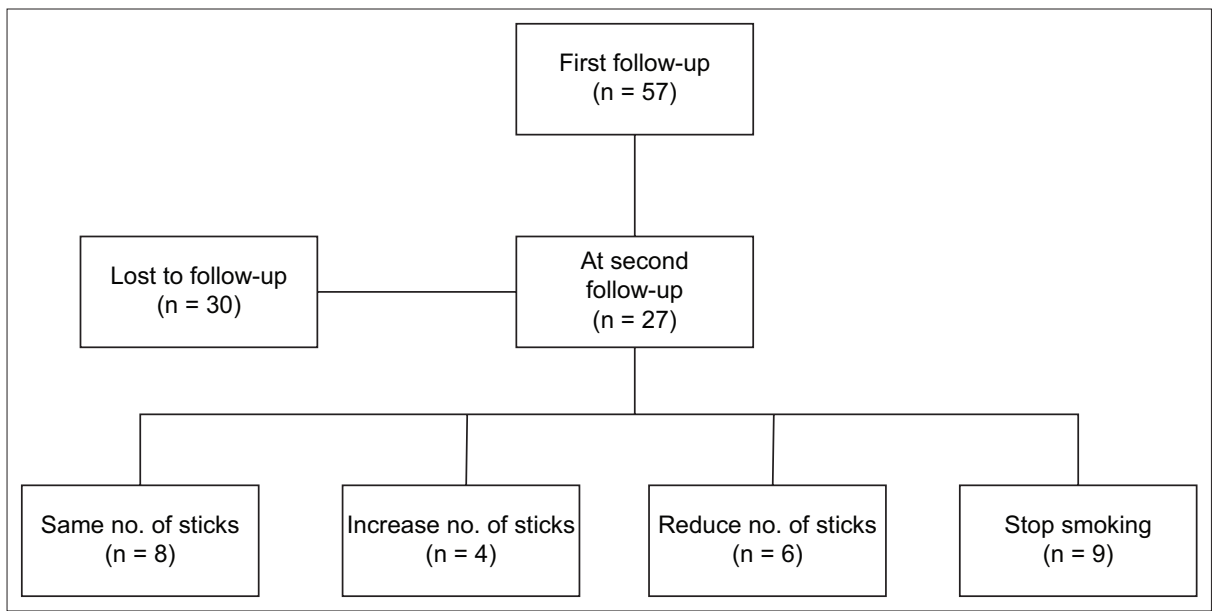

Fig. 3 Diagram shows the change in the number of cigarettes sticks smoked per day at the second follow-up at three months.

characteristics, including current age, age at onset of smoking or smoking history in pack-years. None of these patients had reported any adverse effects from NRT.

A previous study of our local population was performed in 2004. ${ }^{(10)}$ With a larger study population comprising 425 inpatients and 394 outpatients, the authors found that counselling sessions by a psychologist, combined with NRT, achieved a quit rate of $32 \%$ and $36 \%$ among inpatients and outpatients, respectively. ${ }^{(10)}$ The type of NRT and number of counselling sessions did not affect the quit rate at 12 months. ${ }^{(10)}$ Direct comparison of this previous study with our results was not possible due to fundamental differences in the study populations and methods. However, both studies indicated the importance of having trained professionals combined with pharmacotherapy to achieve smoking cessation goals.

To the best of our knowledge, this was the first study examining smoking cessation rates in a dermatological clinic. Our results demonstrate the effectiveness of pharmacist-led smoking cessation counselling in the reduction of smoking rates. Given the adverse effects of smoking on the skin and the importance of stressing on physical appearance for behavioural modification, dermatological clinics represent a unique opportunity and effective platform for clinicians to engage patients in the cessation or reduction of cigarette smoking. Smoking cessation could thus be made a part of the holistic management of dermatological conditions and further efforts should be continued to expand this service to other subspecialties and improve its effectiveness.

\section{REFERENCES}

1. Gill JF, Yu SS, Neuhaus IM. Tobacco smoking and dermatologic surgery. J Am Acad Dermatol 2013; 68:167-72.

2. Gyger G, Hudson M, Lo E, et al. Does cigarette smoking mitigate the severity of skin disease in systemic sclerosis? Rheumatol Int 2013; 33:943-8

3. Ortiz A, Grando SA. Smoking and the skin. Int J Dermatol 2012; 51:250-62.

4. Aubin HJ, Luquiens A, Berlin I. Pharmacotherapy for smoking cessation: pharmacological principles and clinical practice. Br J Clin Pharmacol 2014; 77:324-36.

5. Flett K, Clark-Carter D, Grogan S, Davey R. How effective are physical appearance interventions in changing smoking perceptions, attitudes and behaviours? A systematic review. Tob Control 2013; 22:74-9.

6. Kng KK, Lauw XT, Tan AS, Earnest A. Effectiveness of smoking cessation services in Tan Tock Seng Hospital, Singapore. Ann Acad Med Singapore 2012; 41:230-2.

7. Stead LF, Koilpillai P, Lancaster T. Additional behavioural support as an adjunct to pharmacotherapy for smoking cessation. Cochrane Database Syst Rev 2015; (10):CD009670.

8. Lally $\mathrm{P}$, van Jaarsveld $\mathrm{CH}$, Potts $\mathrm{HW}$, Wardle J. How are habits formed: modelling habit formation in the real world. Eur J Soc Psychol 2010; 40:998-1009.

9. Fildes EE, Kapella-Mshigeni S, Campbell-Heider N. Outcomes of a one-time telephone intervention for smoking cessation in adults. J Addict Nurs 2015; 26:184-90.

10. Zow HC, Hsu AA, Eng PC. Smoking cessation programme: the Singapore General Hospital experience. Singapore Med J 2004; 45:430-4.

11. Thompson TP, Greaves CJ, Ayres R, et al. Factors associated with study attrition in a pilot randomised controlled trial to explore the role of exercise-assisted reduction to stop (EARS) smoking in disadvantaged groups. Trials 2016; 17:524. 